\title{
Computationally Efficient Direction-of-Arrival Estimation Based on Partial A Priori Knowledge of Signal Sources
}

\author{
Lei Huang, ${ }^{1}, 2$ Shunjun Wu, ${ }^{1}$ Dazheng Feng, ${ }^{1}$ and Linrang Zhang ${ }^{1}$ \\ ${ }^{1}$ National Key Laboratory for Radar Signal Processing, Xidian University, 710071 Xi'an, China \\ ${ }^{2}$ Department of Electrical and Computer Engineering, Duke University, Durham, NC 27708-0291, USA
}

Received 19 January 2005; Revised 20 September 2005; Accepted 25 October 2005

Recommended for Publication by Peter Handel

A computationally efficient method is proposed for estimating the directions-of-arrival (DOAs) of signals impinging on a uniform linear array (ULA), based on partial a priori knowledge of signal sources. Unlike the classical MUSIC algorithm, the proposed method merely needs the forward recursion of the multistage Wiener filter (MSWF) to find the noise subspace and does not involve an estimate of the array covariance matrix as well as its eigendecomposition. Thereby, the proposed method is computationally efficient. Numerical results are given to illustrate the performance of the proposed method.

Copyright @ 2006 Hindawi Publishing Corporation. All rights reserved.

\section{INTRODUCTION}

It is desired to estimate the directions-of-arrival (DOAs) of incident signals from noisy data in many areas such as communication, radar, sonar, and geophysical seismology [1]. The classical subspace-based methods, for example, the MUSIC-type [2] algorithms that rely on the decomposition of the observation space into signal subspace and noise subspace, can provide high-resolution DOA estimates with good estimation accuracy. Normally, the classical subspace-based methods are developed without considering any knowledge of the incident signals, except for some general statistical properties like the second-order ergodicity. Nevertheless, the subspace-based methods typically involve the eigendecomposition of the array covariance matrix. As a result, these methods are rather computationally intensive, especially for large arrays.

To attain better DOA estimation accuracy and, perhaps, reduce the computational complexity, a number of algorithms that assume some a priori knowledge, such as the waveforms, of the incident signals have been developed in [3-9]. The assumption is reasonable in friendly communications, such as wireless communications and GPS, where certain a priori knowledge of the incident signals is available to the receiver. The a priori information may or may not be explicit. For example, in a packet radio communication system or a mobile communication system, a known preamble may be added to the message for training purposes. In a digital communication system, the modulation format of the transmitted symbol stream is known to the receiver, although the actual transmitted symbol stream is unknown [10]. With the assumption that the waveforms of the incident signals are known, several computationally efficient maximum likelihood (ML) estimators, for example, the methods named DEML [3], CDEML [4], and WDEML [5] were presented for DOA estimation. Using the known waveforms of the signals, these methods decouple the multidimensional nonlinear optimization of the exact ML estimator to a set of one-dimensional (1D) optimization and, thereby, are relatively computationally simple. To reduce the computational complexity, several algorithms for DOA estimation have been developed by exploiting the partial a priori knowledge of signal sources such as the special features of cyclostationary signals [6] and constant modulus (CM) signals [7]. The authors in [6] utilized the cyclic correlation matrix to calculate the noise subspace through a linear operation. Since this method can avoid the eigendecomposition of the covariance matrix, it is computationally efficient. With the $\mathrm{CM}$ assumption [7], it is possible to find the estimate of the array response matrix, and then use a scheme similar to the ESPRIT method to directly achieve the DOA estimation, therefore avoiding the $1 \mathrm{D}$ search and reducing the computational complexity. Nevertheless, these methods are suitable only for signals with the appropriate special temporal properties. Recently, the reduced-order correlation kernel estimation technique (ROCKET) [11] and ROCK MUSIC algorithms $[8,9]$ were applied to high-resolution spectral 
estimation. Exploiting the received signal of the first array element to initialize the multistage Wiener filter (MSWF) [12], the ROCKET algorithm only needs the forward recursion of the MSWF to find a subspace of interest and use that subspace to calculate a reduced-rank data matrix and a reducedrank weight vector for a reduced-rank autoregressive (AR) spectrum estimator. Given the direction or spatial frequency of one signal, the ROCK MUSIC method can find a nonunitary basis for the signal subspace by using the forward and backward recursions of the MSWF. The ROCKET and ROCK MUSIC algorithms do not resort to the eigendecomposition of the array covariance matrix, giving them a computational advantage. Nevertheless, the ROCK MUSIC algorithm still needs the forward and backward recursions of the MSWF, which increases the complexity of the algorithm since the backward recursion coefficients completely change with each new stage that is added. To find the reduced-rank data matrix and the reduced-rank AR weight vector, the ROCKET method still involves complex matrix-matrix products, implying that additional computational cost is incurred.

In this paper, we propose a computationally efficient method for DOA estimation, based on partial a priori knowledge of signal sources. Using the orthogonal property of the matched filters of the MSWF, we show that the signal subspace and the noise subspace can be spanned by the matched filters. The estimated noise subspace is then exploited to super-resolve the incident signals instead of using the eigendecomposition-based MUSIC method, thus reducing the computational complexity of calculating the noise subspace. To cure coherent signals, we apply the spatial smoothing technique merely to the array data matrix and the training data vector, and therefore avoid the estimate of the array covariance matrix. Unlike the ROCKET and ROCK MUSIC techniques, the proposed method merely needs the forward recursion of the MSWF to obtain the noise subspace and does not require any complex matrix-matrix products, thereby further reducing the computational complexity of the algorithm. Compared to the classical MUSIC estimator and the fast subspace decomposition (FSD) method [13], the proposed method does not involve the estimate of the array covariance matrix or any eigendecomposition. Thus, the novel method is computationally attractive and can be used in the case of small samples where the array covariance matrix cannot be estimated efficiently. While operationally similar to the classical MUSIC estimator, the proposed method finds the noise subspace in a more computationally efficient way, which is the distinguishing feature of the new method.

This paper is organized as follows. Section 2 gives the data model and reviews the MSWF. Section 3 presents the new method for DOA estimation. In Section 4, numerical results are given. Finally, conclusions are drawn in Section 5.

\section{PROBLEM FORMULATION}

\subsection{Data model}

Consider a uniform linear array (ULA) composed of $M$ isotropic sensors. Impinging upon the ULA are $P$ narrowband signals from distinct directions $\theta_{1}, \theta_{2}, \ldots, \theta_{P}$. The $M \times 1$ vector received by the array at the $k$ th snapshot can be expressed as

$$
\mathbf{x}(k)=\sum_{i=1}^{P} \mathbf{a}\left(\theta_{i}\right) s_{i}(k)+\mathbf{n}(k), \quad k=0,1, \ldots, N-1,
$$

where $s_{i}(k)$ is the scalar complex waveform referred to as the $i$ th signal, $\mathbf{n}(k) \in \mathbb{C}^{M \times 1}$ is the additive noise vector, $N$ and $P$ denote the number of snapshots and the number of signals, respectively, $\mathbf{a}\left(\theta_{i}\right)$ is the steering vector of the array toward direction $\theta_{i}$ and takes the following form:

$$
\mathbf{a}\left(\theta_{i}\right)=\left[1, e^{j \varphi_{i}}, \ldots, e^{j(M-1) \varphi_{i}}\right]^{T},
$$

where $\varphi_{i}=(2 \pi d / \lambda) \sin \theta_{i}$ in which $\theta_{i} \in(-\pi / 2, \pi / 2), d$ and $\lambda$ are the interelement spacing and the wavelength, respectively, and the superscript $(\cdot)^{T}$ denotes the transpose operator. Assume that the first signal is the desired signal whose waveform or training data is known.

In matrix form, (1) becomes

$$
\mathbf{x}(k)=\mathbf{A}(\boldsymbol{\theta}) \mathbf{s}(k)+\mathbf{n}(k), \quad k=0,1, \ldots, N-1,
$$

where

$$
\begin{aligned}
\mathbf{A}(\boldsymbol{\theta}) & =\left[\mathbf{a}\left(\theta_{1}\right), \mathbf{a}\left(\theta_{2}\right), \ldots, \mathbf{a}\left(\theta_{P}\right)\right], \\
\mathbf{s}(k) & =\left[s_{1}(k), s_{2}(k), \ldots, s_{P}(k)\right]^{T}
\end{aligned}
$$

are the $M \times P$ steering matrix and the $P \times 1$ complex signal vector, respectively. Throughout this paper, we assume that $M>P$. Furthermore, the background noise uncorrelated with the signals is modeled as a stationary, spatiallytemporally white, zero-mean, complex Gaussian random process.

\subsection{Multistage Wiener filter}

It is well known that the Wiener filter (WF) $\mathbf{w}_{w f} \in \mathbb{C}^{M \times 1}$ can be used to estimate the desired signal $d(k) \in \mathbb{C}$ from the array data $\mathbf{x}(k)$ in the minimum mean square error (MMSE) sense. Thereby, we have the following design criterion:

$$
\mathbf{w}_{w f}=\arg \min _{\mathbf{w}} E\left\{\left|d(k)-\mathbf{w}^{H} \mathbf{x}(k)\right|^{2}\right\},
$$

where $\hat{d}(k)=\mathbf{w}^{H} \mathbf{x}(k)$ represents the estimate of the desired signal $d(k)$, and $\mathbf{w} \in \mathbb{C}^{M \times 1}$ is the linear filter. Solving (5) leads to the Wiener-Hopf equation

$$
\mathbf{R}_{\mathbf{x}} \mathbf{w}_{w f}=\mathbf{r}_{\mathbf{x} d}
$$

where $\mathbf{R}_{\mathbf{x}}=E\left[\mathbf{x}(k) \mathbf{x}^{H}(k)\right], \mathbf{r}_{\mathbf{x} d}=E\left[\mathbf{x}(k) d^{*}(k)\right]$. The classical Wiener filter, that is, $\mathbf{w}_{w f}=\mathbf{R}_{\mathbf{x}}^{-1} \mathbf{r}_{\mathbf{x} d}$, is computationally intensive for large $M$ since the inverse of the array covariance matrix $\mathbf{R}_{\mathbf{x}}$ is involved. The MSWF developed by Goldstein et al. [12] is to find an approximate solution to the WienerHopf equation, which does not need the inverse of the array covariance matrix. The MSWF of rank $D$ based on the datalevel lattice structure [14] is shown in Algorithm 1. 
Figure 1 illustrates the lattice structure of the MSWF. The reference signal $d_{0}(k)$ is the training data of the desired signal, which is available in friendly communications. In this paper, let $d_{0}(k)=s_{1}(k)$. The observation data $\mathbf{x}_{i-1}(k)$ at the $i$ th stage are partitioned into an interesting signal $d_{i}(k)$ and its orthogonal component $\mathbf{x}_{i}(k)$. The desired signal $d_{i}(k)$ is obtained by prefiltering $\mathbf{x}_{i-1}(k)$ with the matched filters $\mathbf{h}_{i}$, but is annihilated by the blocking matrix $\mathbf{B}_{i}=\mathbf{I}-\mathbf{h}_{i} \mathbf{h}_{i}^{H}$. The array data matrix is partitioned stage-by-stage in the same manner. As a result, we can readily achieve the prefiltering $\operatorname{matrix} \mathbf{T}_{M}=\left[\mathbf{h}_{1}, \mathbf{h}_{2}, \ldots, \mathbf{h}_{M}\right]$.

\section{COMPUTATIONALLY EFFICIENT ALGORITHM FOR DOA ESTIMATION}

It is shown in [15] that all the matched filters $\mathbf{h}_{i}, i=$ $1,2, \ldots, D(D \leq P)$ are contained in the column space of $\mathbf{A}(\boldsymbol{\theta})$ by assuming $d_{0}(k)=s_{1}(k)$. It follows that the orthogonal matched filters $\mathbf{h}_{1}, \mathbf{h}_{2}, \ldots, \mathbf{h}_{P}$ span the signal subspace, namely,

$$
\operatorname{span}\left\{\mathbf{h}_{1}, \mathbf{h}_{2}, \ldots, \mathbf{h}_{P}\right\}=\operatorname{col}\{\mathbf{A}(\boldsymbol{\theta})\}
$$

Since all the matched filters $\mathbf{h}_{1}, \mathbf{h}_{2}, \ldots, \mathbf{h}_{M}$ are mutually orthogonal for the special choice of the blocking matrix $\mathbf{B}_{i}=$ $\mathbf{I}-\mathbf{h}_{i} \mathbf{h}_{i}^{H}$, the matched filters after the Pth stage of the MSWF are orthogonal to the signal subspace, that is, $\mathbf{h}_{i} \perp \operatorname{col}\{\mathbf{A}(\boldsymbol{\theta})\}$ for $i=P+1, P+2, \ldots, M$. Therefore, the last $M-P$ matched filters span the orthogonal complement of the signal subspace, namely the noise subspace:

$$
\operatorname{span}\left\{\mathbf{h}_{P+1}, \mathbf{h}_{P+2}, \ldots, \mathbf{h}_{M}\right\}=\operatorname{null}\{\mathbf{A}(\boldsymbol{\theta})\} .
$$

Equation (8) indicates that the noise subspace can be readily obtained by performing the forward recursion of the MSWF, and thus the MUSIC estimator based on the noise subspace can be exploited to produce peaks at the DOA locations. For coherent signals, however, the noise subspace estimated by this method is no longer correct. That is to say, the last $M-P$ matched filters do not span a noise subspace for the case where the signals are coherent. As a result, we must resort to the smoothing techniques to decorrelate the coherent signals. Since the array covariance matrix is not involved in computing the basis vectors for the noise subspace, we perform the spatial smoothing method [16] merely to the array data matrix.

For the spatial smoothing technique, an array consisting of $M$ sensors is subdivided into $L$ subarrays. Thereby, the number of elements per subarray is $M_{L}=M-L+1$. For $l=1,2, \ldots, L$, let the $M_{L} \times M$ matrix $\mathbf{J}_{l}$ be a selection matrix that takes the following form:

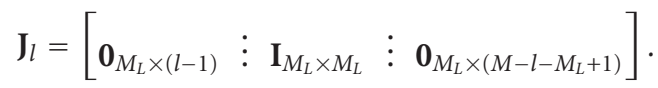

The selection matrix $\mathbf{J}_{l}$ is used to select part of the $M \times N$ array data matrix $\mathbf{X}_{0}=\left[\mathbf{x}_{0}(0), \mathbf{x}_{0}(1), \ldots, \mathbf{x}_{0}(N-1)\right]$, which corresponds to the $l$ th subarray. Hence, the spatially smoothed (i) Initialization. $d_{0}(k)$ and $\mathbf{x}_{0}(k)=\mathbf{x}(k)$.

(ii) Forward recursion. For $i=1,2, \ldots, D$,

$$
\begin{gathered}
\mathbf{h}_{i}=\frac{E\left[\mathbf{x}_{i-1}(k) d_{i-1}^{*}(k)\right]}{\left\|E\left[\mathbf{x}_{i-1}(k) d_{i-1}^{*}(k)\right]\right\|_{2}} ; \\
d_{i}(k)=\mathbf{h}_{i}^{H} \mathbf{x}_{i-1}(k) ; \\
\mathbf{x}_{i}(k)=\mathbf{x}_{i-1}(k)-\mathbf{h}_{i} d_{i}(k)
\end{gathered}
$$

(iii) Backward recursion. For $i=D, D-1, \ldots, 1$ with $e_{D}(k)=d_{D}(k)$,

$$
\begin{gathered}
w_{i}=\frac{E\left[d_{i-1}(k) e_{i}^{*}(k)\right]}{E\left[\left|e_{i}(k)\right|^{2}\right]} ; \\
e_{i-1}(k)=d_{i-1}(k)-w_{i}^{*} e_{i}(k) .
\end{gathered}
$$

Algorithm 1

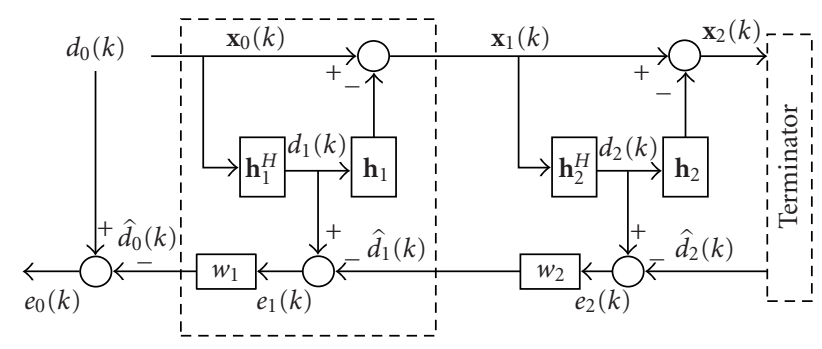

Figure 1: Lattice structure of the MSWF. The dashed line denotes the basic box for each additional stage.

$M_{L} \times L N$ data matrix $\overline{\mathbf{X}}_{0}$ is constructed as

$$
\overline{\mathbf{X}}_{0}=\left[\begin{array}{llll}
\mathbf{J}_{1} \mathbf{X}_{0} & \mathbf{J}_{2} \mathbf{X}_{0} & \cdots & \mathbf{J}_{L} \mathbf{X}_{0}
\end{array}\right] \in \mathbb{C}^{M_{L} \times L N} .
$$

Similarly to the spatially smoothed data matrix $\overline{\mathbf{X}}_{0}$, the "spatially smoothed" training data vector should have the form

$$
\overline{\mathbf{d}}_{0}=[\underbrace{\mathbf{d}_{0} ; \mathbf{d}_{0} ; \cdots ; \mathbf{d}_{0}}_{L}] \in \mathbb{C}^{L N \times 1},
$$

where $\mathbf{d}_{0}=\left[d_{0}(0), d_{0}(1), \ldots, d_{0}(N-1)\right]^{T} \in \mathbb{C}^{N \times 1}$ and ";" denotes vertical concatenation. Accordingly, the $i$ th spatially smoothed matched filter of the MSWF is computed as

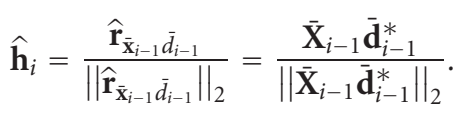

Thus, the computationally efficient algorithm for DOA estimation can be summarized as shown in Algorithm 2.

Remark 1. Notice that the lattice structure of the MSWF avoids the formation of blocking matrices, and all the operations of the MSWF only involve complex vector-vector products. Consequently, the proposed method merely requires $O(M N)$ flops to calculate each basis vector $\mathbf{h}_{i}$ and thereby 
Step 1. Apply the spatial smoothing technique to the $M \times N$ data matrix $\mathbf{X}_{0}$ and obtain the spatially smoothed $M_{L} \times L N$ data matrix $\overline{\mathbf{X}}_{0}$.

Step 2. Construct the spatially smoothed training data vector $\overline{\mathbf{d}}_{0}$ as (12).

Step 3. Perform the following $M_{L}$ recursions.

$$
\begin{aligned}
& \text { For } i=1,2, \ldots, M_{L}, \\
& \qquad \begin{array}{r}
\hat{\mathbf{h}}_{i}=\frac{\overline{\mathbf{X}}_{i-1} \overline{\mathbf{d}}_{i-1}^{*}}{\left\|\overline{\mathbf{X}}_{i-1} \overline{\mathbf{d}}_{i-1}^{*}\right\|_{2}}, \\
\overline{\mathbf{d}}_{i}=\hat{\mathbf{h}}_{i}^{H} \overline{\mathbf{X}}_{i-1}, \\
\overline{\mathbf{X}}_{i}=\overline{\mathbf{X}}_{i-1}-\hat{\mathbf{h}}_{i} \overline{\mathbf{d}}_{i} .
\end{array}
\end{aligned}
$$

Obtain the estimated noise subspace

$$
\hat{\mathbf{N}}_{M_{L}-P}=\left[\hat{\mathbf{h}}_{P+1}, \hat{\mathbf{h}}_{P+2}, \ldots, \hat{\mathbf{h}}_{M_{L}}\right] \text {. }
$$

Step 4. Exploit the MUSIC estimator

$P_{\text {MUSIC }}(\theta)=1 /\left(\mathbf{a}_{M_{L}}^{H}(\theta) \hat{\mathbf{N}}_{M_{L}-P} \hat{\mathbf{N}}_{M_{L}-P}^{H} \mathbf{a}_{M_{L}}(\theta)\right)$ to produce peaks at the DOA locations, where

$\mathbf{a}_{M_{L}}(\theta)=\left(1 / \sqrt{M_{L}}\right)\left[1, e^{j \varphi_{i}}, \ldots, e^{j\left(M_{L}-1\right) \varphi_{i}}\right]^{T}$. Alternatively, the DOAs can also be estimated by the root-MUSIC algorithm: finding the $P$ roots, say $\hat{z}_{1}, \hat{z}_{2}, \ldots, \hat{z}_{P}$ that have the largest magnitude, of the root-MUSIC polynomial

$D(z)=z^{M_{L}-1} \mathbf{p}^{T}\left(z^{-1}\right) \hat{\mathbf{N}}_{M_{L}-P} \hat{\mathbf{N}}_{M_{L}-P}^{H} \mathbf{p}(z)$ where $\mathbf{p}(z)=\left[1, z, \ldots, z^{M_{L}-1}\right]^{T}$, yields the DOA estimates as $\hat{\theta}_{i}=\arcsin \left(\lambda \arg \left(\hat{z}_{i}\right) / 2 \pi d\right)$ in which $\arg \left(\hat{z}_{i}\right)$ denotes the phase angle of the complex number $\hat{z}_{i}$.

\section{Algorithm 2}

needs $O\left(M^{2} N\right)$ flops to obtain the noise subspace for the case of uncorrelated signals. Additionally, this method does not rely on the eigendecomposition of the array covariance matrix, saving the computational cost of $O\left(M^{3}\right)$. Thus, the proposed method is more computationally efficient than the classical MUSIC algorithm, especially for large $M$.

Remark 2. It should be noted that the proposed method can determine the directions of the desired signal with the knowledge of training data and the interferences without the knowledge of training data. That is to say, the proposed method only needs partial a priori knowledge of signal sources, such as the training data of the desired signal, to estimate the DOAs of all the incident signals.

\section{NUMERICAL RESULTS}

\subsection{Uncorrelated signals}

Assume that there are two uncorrelated signals with equal power impinging upon the ULA composed of 10 sensors from directions $\left\{0^{\circ}, 5^{\circ}\right\}$, and that signal 1 is the desired signal whose waveform is known a priori. We also assume that the number of signals is known. The background noise is a sta-

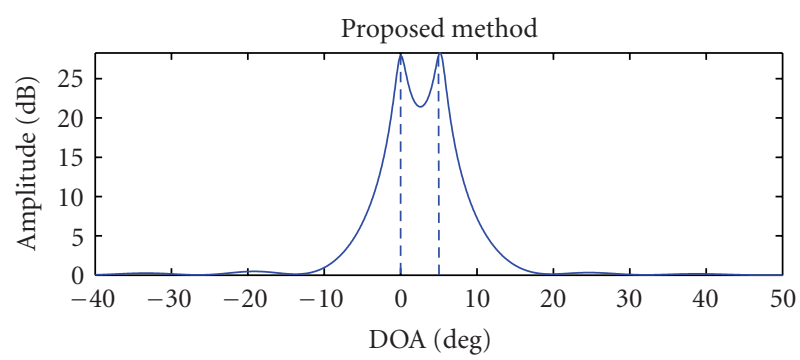

(a)

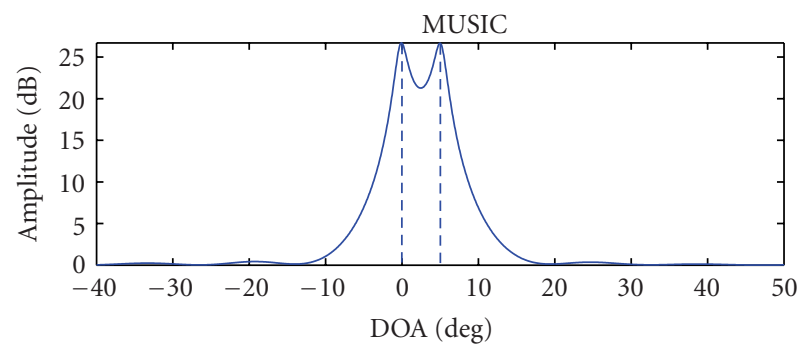

(b)

FIGURE 2: Spatial spectra of uncorrelated signals based on one trial. $N=64, M=10$, and SNR $=10 \mathrm{~dB}$. The vertical dashed line denotes the true locations of incident signals.

tionary, spatially-temporally white, complex Gaussian random process with zero-mean and the variance $\sigma_{\mathbf{n}}^{2}$.

The spatial spectra of the proposed method and the classical MUSIC algorithm are shown in Figure 2, where $N=64$ and signal-to-noise ratio (SNR) is $10 \mathrm{~dB}$. SNR is defined as $10 \log \left(\sigma_{\mathrm{s}}^{2} / \sigma_{\mathrm{n}}^{2}\right)$, where $\sigma_{\mathrm{s}}^{2}$ is the power of each signal in a single sensor. From Figure 2, it can be observed that the proposed method works very much like the classical MUSIC algorithm. To evaluate the estimation performance of the proposed method, we exploit the root-MUSIC algorithm to yield the DOAs of the incident signals and make 500 Monte Carlo runs to compute the root-mean-squared errors (RMSEs) of estimated DOAs. The RMSEs of estimated DOAs versus SNR are shown in Figure 3, where $N=64$. The CramérRao bounds (CRBs) [17] are also plotted for comparison. As shown in Figure 3, when SNR is lower than $6 \mathrm{~dB}$ the proposed estimator surpasses the classical MUSIC algorithm, especially in the estimation of the first signal since its waveform is known and used to calculate the basis vectors for the noise subspace. As SNR increases, the proposed method provides the same estimation accuracy as the classical MUSIC algorithm. The RMSEs of the two signals for the two methods approach to the corresponding CRBs when SNR becomes high. The RMSEs of the estimated DOAs for the two methods versus the number of snapshots are demonstrated in Figure 4, where $\mathrm{SNR}=5 \mathrm{~dB}$. It can be observed from Figure 4 that the estimation accuracy of the proposed method is higher than that of the classical MUSIC estimator when the number of snapshots is less than 64 . As the samples become large, the proposed method yields the same estimation accuracy as the classical MUSIC method. 


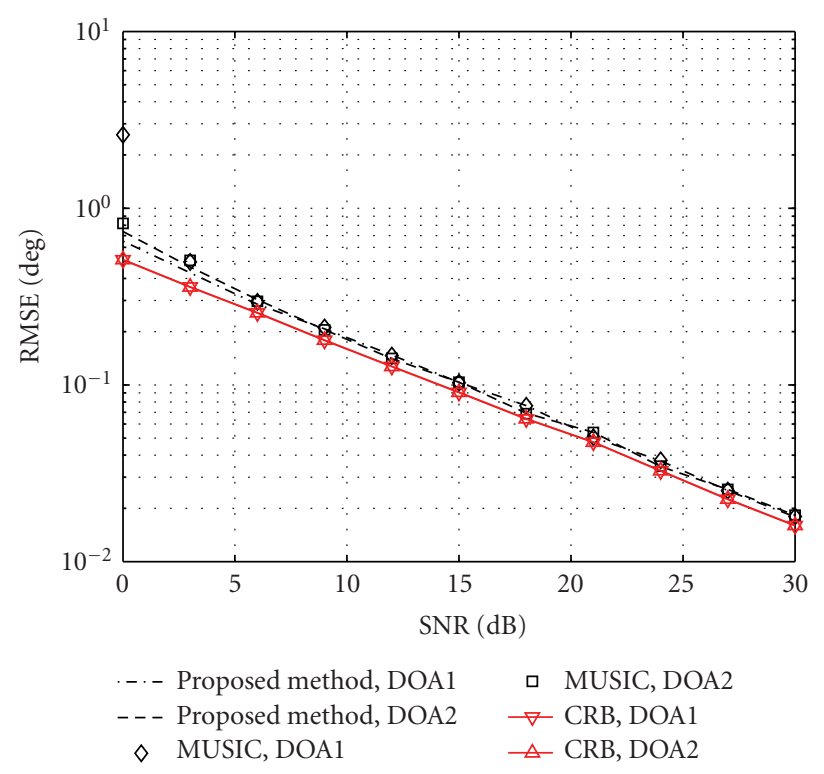

FIGURE 3: RMSE of estimated DOA for uncorrelated signals versus SNR. $N=64$ and $M=10$.

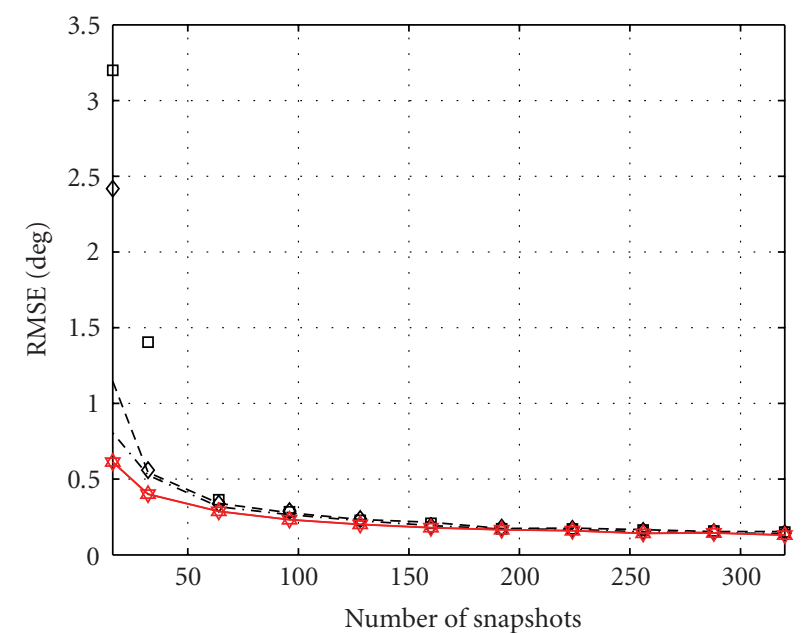

$$
\begin{aligned}
& \text {-. - Proposed method, DOA1 } \square \text { MUSIC, DOA2 } \\
& \text { - - - Proposed method, DOA2 } \quad \nabla \text { CRB, DOA1 } \\
& \diamond \quad \text { MUSIC, DOA1 } \quad \triangle \text { CRB, DOA2 }
\end{aligned}
$$

FIGURE 4: RMSE of estimated DOA for uncorrelated signals versus number of snapshots. SNR $=5 \mathrm{~dB}$ and $M=10$.

\subsection{Coherent signals}

Consider the case where there are two signals impinging upon the ULA consisting of 12 sensors from the same signal source whose waveform is known a priori. The first is a direct-path signal and the other refers to the scaled and delayed replicas of the first signal that represent the multipaths or the "smart" jammers. The propagation constants are

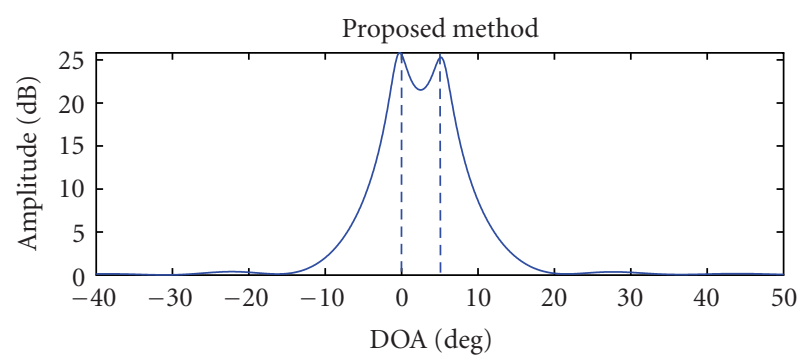

(a)

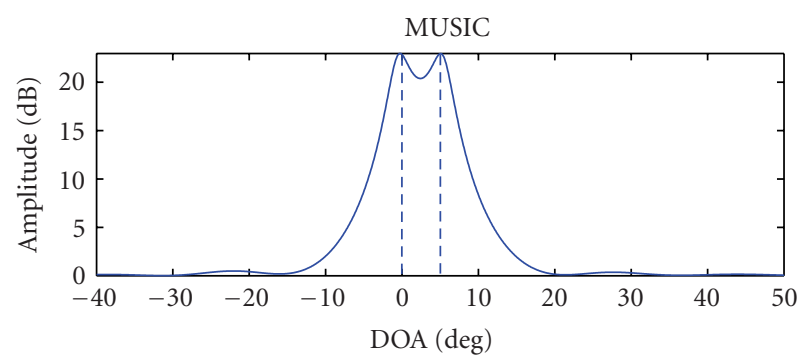

(b)

FIGURE 5: Spatial spectra of coherent signals based on one trial. $N=$ $64, M=12, M_{L}=9$, and SNR $=10 \mathrm{~dB}$. The vertical dashed line denotes the true locations of incident signals.

$\{1,-0.8+j 0.6\}$. We assume that the true DOAs are $\left\{0^{\circ}, 5^{\circ}\right\}$ and the number of signals is known. The background noise is identical to that in the case of uncorrelated signals. To decorrelate the incident coherent signals, the spatial smoothing technique is also applied to the classical MUSIC algorithm.

The spatial spectra of the proposed method and the classical MUSIC algorithm are shown in Figure 5, where $N=64$, $\mathrm{SNR}=10 \mathrm{~dB}$, and the number of sensors of the subarray is 9 , namely $M_{L}=9$. Figure 5 indicates that the proposed estimator works very much like the classical MUSIC estimator in the case of coherent signals. The following results are based on 500 Monte Carlo trials. The RMSEs of estimated DOAs versus SNR are shown in Figure 6, where $N=64$. For comparison, the CRBs [18] for coherent signals are given as well. From Figure 6, it can be observed that the proposed method clearly outperforms the classical MUSIC algorithm when SNR $\leq 6 \mathrm{~dB}$, and provides the same estimation accuracy as the latter when SNR $>6 \mathrm{~dB}$. The RMSEs of estimated DOAs for the two methods versus the number of snapshots are plotted in Figure 7, where SNR $=5 \mathrm{~dB}$. It is shown in Figure 7 that the proposed method surpasses the classical MUSIC estimator when the number of snapshots is less than 96 and provides the same estimation accuracy as the latter when the samples become large. Since the waveform of the desired signal is known and exploited to compute the new basis vectors for the signal subspace and the noise subspace, the new signal subspace is capable of capturing the signal information while excluding a large portion of the noise. On the contrary, its orthogonal complement can eliminate the signals more accurately from the noisy data and, thereby, is a 


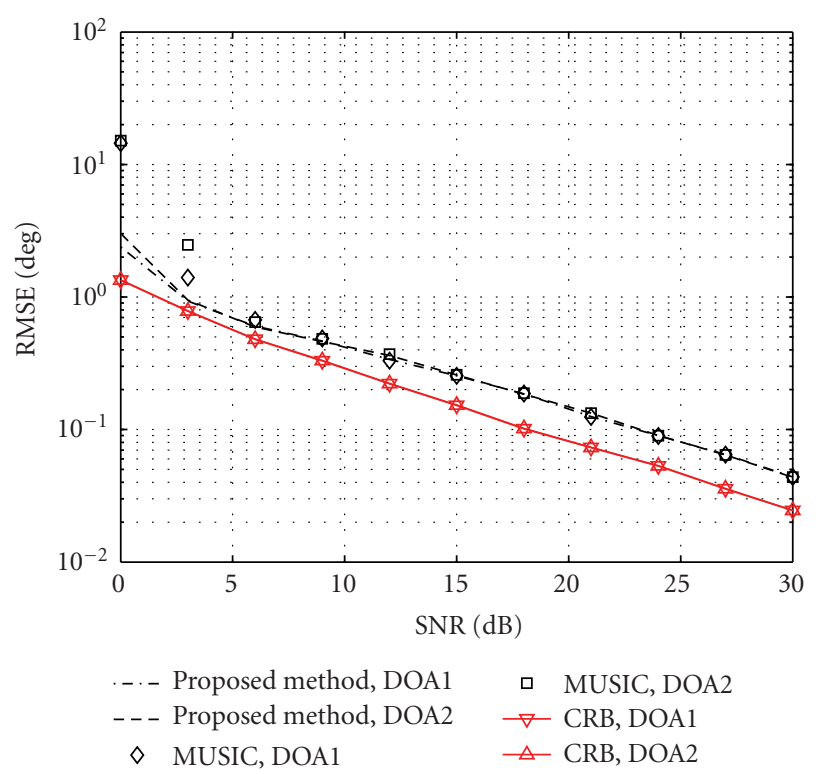

FIGURE 6: RMSE of estimated DOA for coherent signals versus SNR. $N=64, M=12$, and $M_{L}=9$.

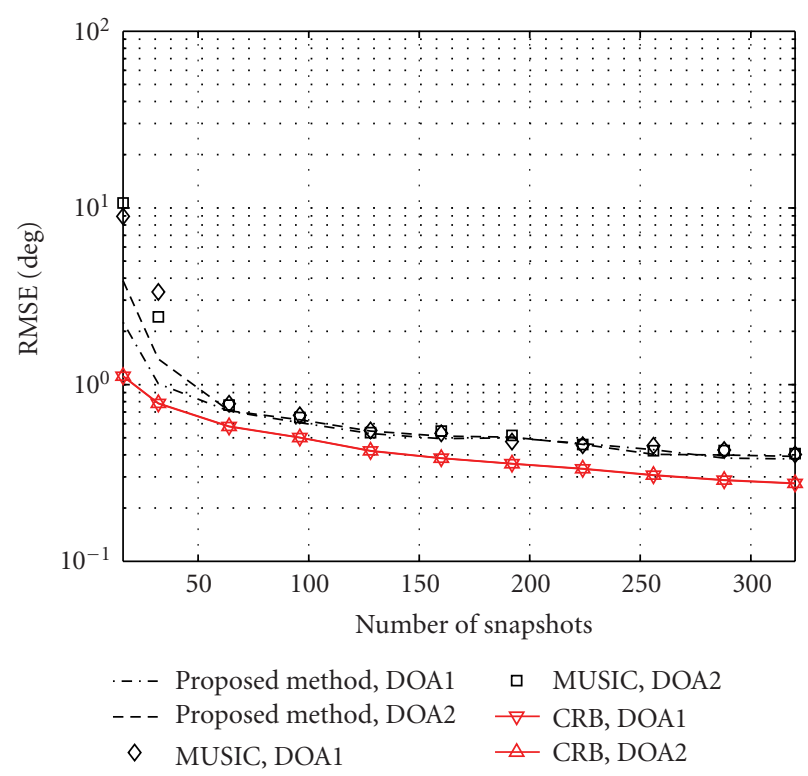

FIgURE 7: RMSE of estimated DOA for coherent signals versus number of snapshots. $\mathrm{SNR}=5 \mathrm{~dB}, M=12$, and $M_{L}=9$.

cleaner noise subspace that leads to the enhanced estimation performance.

\section{CONCLUSION}

We have presented a computationally efficient method for DOA estimation in this paper. The proposed method only needs the forward recursion of the MSWF and does not resort to the eigendecomposition of the array covariance ma- trix, thereby requiring lower computational cost than the classical MUSIC algorithm especially in the case of a large array. Numerical results indicate that the proposed method surpasses the classical MUSIC estimator for the case of small samples and/or low SNR and provide the same estimation performance as the latter when the samples become large and/or SNR increases.

\section{REFERENCES}

[1] P. Stoica and R. Moses, Introduction to Spectral Analysis, Prentice-Hall, Upper Saddle Revier, NJ, USA, 1997.

[2] R. O. Schmidt, A signal subspace approach to multiple emitter location and spectral estimation, Ph.D. thesis, Stanford University, Stanford, Calif, USA, November 1981.

[3] J. Li, B. Halder, P. Stoica, and M. Viberg, "Computationally efficient angle estimation for signals with known waveforms," IEEE Transactions on Signal Processing, vol. 43, no. 9, pp. 21542163, 1995.

[4] M. Cedervall and R. L. Moses, "Efficient maximum likelihood DOA estimation for signals with known waveforms in the presence of multipath," IEEE Transactions on Signal Processing, vol. 45, no. 3, pp. 808-811, 1997.

[5] H. Li, H. Pu, and J. Li, "Efficient maximum likelihood angle estimation for signals with known waveforms in white noise," in Proceedings of 9th IEEE Signal Processing Workshop on Statistical Signal and Array Processing, pp. 25-28, Portland, Ore, USA, September 1998.

[6] J. Xin, H. Tsuji, H. Ohmori, and A. Sano, "Directions-ofarrival tracking of coherent cyclostationary signals without eigendecomposition," in Proceedings of 3rd IEEE Workshop on Signal Processing Advances in Wireless Communications (SPAWC '01), pp. 318-321, Taoyuan, Taiwan, March 2001.

[7] A. Leshem and A.-J. van der Veen, "Direction-of-arrival estimation for constant modulus signals," IEEE Transactions on Signal Processing, vol. 47, no. 11, pp. 3125-3129, 1999.

[8] H. E. Witzgall and J. S. Goldstein, "ROCK MUSIC-nonunitary spectral estimation,” Tech. Rep. ASE-00-05-001, SAIC, May 2000.

[9] H. E. Witzgall, J. S. Goldstein, and M. D. Zoltowski, "A nonunitary extension to spectral estimation," in Proceedings of 9th IEEE Digital Signal Processing Workshop (DSP '00), Hunt, Tex, USA, October 2000.

[10] J. Ward and R. T. Compton Jr., "Improving the performance of a slotted ALOHA packet radio network with an adaptive array," IEEE Transactions on Communications, vol. 40, no. 2, pp. 292-300, 1992.

[11] H. E. Witzgall and J. S. Goldstein, "Detection performance of the reduced-rank linear predictor ROCKET," IEEE Transactions on Signal Processing, vol. 51, no. 7, pp. 1731-1738, 2003.

[12] J. S. Goldstein, I. S. Reed, and L. L. Scharf, "A multistage representation of the Wiener filter based on orthogonal projections," IEEE Transactions on Information Theory, vol. 44, no. 7, pp. 2943-2959, 1998.

[13] G. Xu and T. Kailath, "Fast subspace decomposition," IEEE Transactions on Signal Processing, vol. 42, no. 3, pp. 539-551, 1994.

[14] D. Ricks and J. S. Goldstein, "Efficient implementation of multi-stage adaptive Wiener filters," in Proceedings of Antenna Applications Symposium, Allerton Park, Ill, USA, September 2000. 
[15] L. Huang, S. Wu, D. Feng, and L. Zhang, "Low complexity method for signal subspace fitting," Electronics Letters, vol. 40, no. 14, pp. 847-848, 2004.

[16] T.-J. Shan, M. Wax, and T. Kailath, "On spatial smoothing for direction-of-arrival estimation of coherent signals," IEEE Transactions on Acoustics, Speech, and Signal Processing, vol. 33, no. 4, pp. 806-811, 1985.

[17] P. Stoica and A. Nehorai, "MUSIC, maximum likelihood, and Cramer-Rao bound," IEEE Transactions on Acoustics, Speech, and Signal Processing, vol. 37, no. 5, pp. 720-741, 1989.

[18] A. J. Weiss and B. Friedlander, "On the Cramer-Rao bound for direction finding of correlated signals," IEEE Transactions on Signal Processing, vol. 41, no. 1, pp. 495-499, 1993.

Lei Huang was born in Guangdong, China. He received the B.E., M.E., and Ph.D. degrees in electronic engineering from Xidian University, Xi'an, China, in 2000, 2003, and 2005, respectively. From 2002 to 2005, he was with the National Key Laboratory for Radar Signal Processing, Xidian University, where he worked on signal processing, adaptive filtering, and their applications in wireless communication systems. Since May

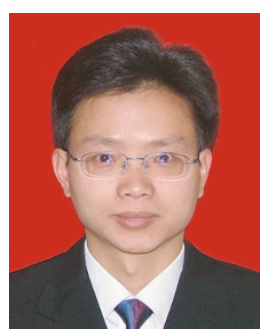
2005, he has been working as a Research Associate in the Department of Electrical and Computer Engineering, Duke University, Durham, NC. His current research interests are statistical signal processing, physical-based signal processing, remote sensing, array processing, and adaptive filtering.

Shunjun Wu was born in Shanghai, China, on February 18, 1942. He graduated from Xidian University in 1964, and since then joined the faculty of the Department of Electrical Engineering, Xidian University. From 1981 to 1983 , he has been a Visiting Scholar in the Department of Electrical Engineering, University of Hawaii at Manoa, USA. He is a Professor at Xidian University and a Senior Member of the Chinese Insti-

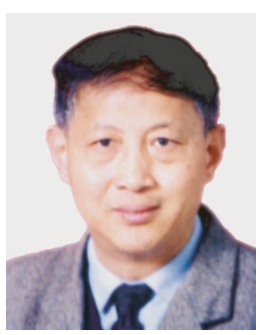
tute of Electronics (CIE). He is currently the Director of the Electronic Engineering Research Institute, Xidian University. His research interests include digital signal processing, adaptive filter, and multidimensional signal processing with applications to radar systems.

Dazheng Feng was born in December 1959. He graduated from Xi'an University of Technology, Xi'an, China, in 1982. He received the M.S. degree from Xi'an Jiaotong University in 1986, and the Ph.D. degree in electronic engineering in 1995 from Xidian University, Xi'an, China. From May 1996 to May 1998, he was a Postdoctoral Research Affiliate and an Associate Professor at Xi' an Jiaotong University, China. From May 1998

to June 2000, he was an Associate Professor at Xidian University. Since July 2000, he has been a Professor at Xidian University. He has published more than 40 journal papers. His research interests include signal processing, intelligence information processing, and InSAR.

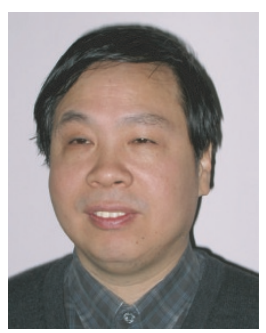

Linrang Zhang was born in Shaanxi province, China. He received his B.E., M.E., and $\mathrm{Ph} . \mathrm{D}$. degrees in electrical engineering from Xidian University, China, in 1988, 1991, and 1999, respectively. From 1991 to present, he has been with the National Key Laboratory of Radar Signal Processing, Xidian University, where he is currently a Professor. He was a Visiting Scholar at the City University of Hong Kong during 2002-2003. His ma-

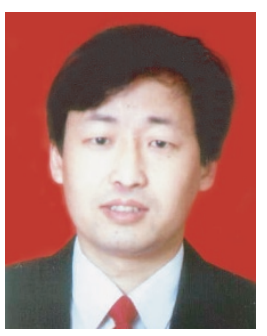
jor research interests have been statistical signal processing, array signal processing, smart antenna, and radar system design. He is a Member of IEEE. 